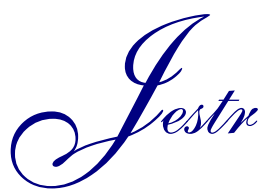

Journal of Engineering Science and Technology Review 12 (5) (2019) 43 - 52

Research Article

\title{
Self-starting Characteristics of Magnetic Controlled Spiral Capsule Robots
}

www.jestr.org

\author{
Jingcai Bai", Junxiao Wu, Minglu Chi, Zheng Fan and Zhiyong Du \\ School of Electrical Engineering and Automation, Henan Institute of Technology, Xinxiang 453003, China
}

Received 17 July 2019; Accepted 21 September 2019

\begin{abstract}
The low power consumption and operability of magnetic controlled spiral capsule robots (MCSCRs) are important indexes that influence the interventional diagnosis and treatment of digestive tract. However, existing studies lack of analysis on the self-starting characteristics of MCSCRs. For simple and effective swimming of a MCSCR in a liquid environment, a self-starting characteristic model of MCSCR was proposed. A new portable electromagnetic propulsion device with dynamic rotating magnetic field and a MCSCR were also developed. A spatial magnetic field distribution model of a permanent magnet was constructed in accordance with the Biot-Savart law. This model was employed to determine the coupling characteristic relation between master and slave permanent magnets. A self-starting model of robots in a pipe filled with liquid was deduced, and the interaction laws of self-starting rotating speed, fluid viscosity, and axial thrust were determined. Results demonstrate that the self-starting rotating speed of robots increases with the increase in fluid viscosity, and the axial thrust is enhanced gradually as rotating speed increases. When the fluid viscosity is $0.1 \mathrm{~Pa} \cdot \mathrm{s}$, the self-starting rotating speed reaches the minimum value $(85 \mathrm{r} / \mathrm{min})$. Self-starting control of robots with a low torque and a strong axial thrust can be realized by adjusting the self-starting distance and rotating speed between the master permanent magnet and robot. When the master permanent magnet is $29 \mathrm{~mm}$ away from the robot, the maximum coupling magnetic moment is the lowest $(0.1 \mathrm{~Pa} \cdot \mathrm{s})$. This study can provide theoretical supports to low-power rapid medical examination based on in vivo capsule robot.
\end{abstract}

Keywords: Magnetic controlled spiral capsule robot, Self-starting characteristics, Magnetic field distribution, Fluid viscosity

\section{Introduction}

A wireless capsule robot (CR), which is a noninvasive and painless diagnosis and treatment tool for the gastrointestinal tract (GIT) of humans, can improve the examination comfort and tolerance of patients effectively. Under the assistance of such wireless CR, examination in blind spots, including GIT, is feasible [1]. Capsule endoscope offers a noninvasive and anodynia examination mode. Capsule endoscope based on wireless image transmission of an embedded visual system can achieve noninvasive diagnosis and treatment in GIT. The clinical applications of gastrointestinal capsule endoscope have expanded the view of medical diagnosis and treatment. CR was initially mainly applied to examination in small intestine. Subsequently, CR begins to be applied to examination in stomach and large intestine gradually. Representative commercialized capsule products include the PillCam Capsule of Given Imaging Company (Israel), Miro Capsule of Korea, and OMOM Capsule of Chongqing Jinshan Company [2-3].

However, these CR products are mainly passive and uncontrollable. They can only move forward by the peristalsis of GIT and even may cause risks of retention in narrow and wrinkled intestinal canal. Statistics of research results shows that the missing area of CR in GIT is approximately $20 \%$, and CR may remain in intestinal tract,

\footnotetext{
*E-mail address: okbi@@163.com

ISSN: 1791-2377 @ 2019 School of Science, HHU. All rights reserved.

doi:10.25103/iestr.125.06
}

which may cause great damages to the psychological and physical health of patents. Therefore, studying passive CRs is a great challenge.

On this basis, many scholars have studied active CRs, which can realize active adjustment of the direction and posture of robots to decrease missing area. Types of active CRs include paddling type [4], peristaltic type [5], legged type [6], and screw propulsion type [7]. Nevertheless, these robots all use self-load power-driven mode and require installation of complicated driving and transmission mechanisms in addition to a battery. The moving ability of these robots is restricted by the capacity of the battery. Moreover, these robots are difficult to control and minimize [8]. CRs, which are driven by an external magnetic field, can avoid these problems completely; they have the characteristics of simple adjustment of magnetic field, good continuous movement, and easy operation and control. CRs driven by an external magnetic field are unrestricted by the internal space and can be highly minimized. Magnetic controlled spiral CRs (MCSCRs) are therefore applicable to clinics.

From the above-mentioned analysis, a portable electromagnetic propulsion system with dynamic rotating magnetic field and a MCSCR were studied. This system not only is easy to be operated but also can generate a dynamic rotating magnetic field and solve problems concerning singular point of robot arm with six degrees of freedom (DOFs) and complicated operation driven by electromagnetic coil. Starting and stopping of the robot can be controlled simply by the self-starting characteristics. The swimming speed of the MCSCR can be controlled by 
adjusting the rotating speed of the master permanent magnet. The MCSCR is guided to make direct movement and turning by the rotating magnetic field produced by the master permanent magnet. This study provides some references to develop portable MCSCR-based propulsion devices.

\section{State of the art}

The current external magnetic field-driven system can be divided into electromagnetic coil-driven system and permanent magnet-driven system [9-10]. The former generates a magnetic moment by the current input coil and can offer continuous control over magnetic field intensity and frequency. Park [11] and $\mathrm{Fu}$ [12-13] formed an external magnetic field with Helmholtz coils to drive and control a spiral CR. The rotating movement produced by the coil magnetic field could drive the robot to make rotational motion. The direction, frequency, and intensity of the magnetic field were also controllable. Sendoh [14] realized precession of spiral robots by generating plane-rotating magnetic fields with coils but failed to generate spatial rotating magnetic fields. Lee [15] created uniform and gradient magnetic fields by using five pairs of coils to drive the movement, turning, and spiral walking of a robot. However, convenience in magnetic field control was ignored. Guo [16] studied an external magnetic field that was formed by three pairs of round Helmholtz coils and adjusted the direction and moving speed of a robot by changing the direction and frequency of the magnetic field; this way realizes climbing and turning of the robot. Nevertheless, the electromagnetic coil-driven system in clinics requires a large volume of coil to cover the human body. Such system not only induces a high cost but also needs physicians to master complicated calculation and controlling techniques during operation. This condition proposes a great challenge to physicians. Yang [17] designed a wireless energy transmission system for a CR that could offer stable energy supply under any postures, but the new power-supply coils had a complicated structure and large volume. Zhang [18] analyzed the uniformity of a magnetic field and the wireless power supply system based on a magnetic controlled multimodule CR system, but great errors in synchronism and jointing reliability of the multimodule structure at moving occurred. A permanent magnet-driven system forms a coupling magnetic moment by using a permanent magnet or an electromagnet on the robot arm or the end of a portable driving device and the embedded magnet in CR; this way controls the direction, position, and rotating speed of the robot. Salerno [19] and Gumprecht [20] drove a robot by applying a magnetic force or magnetic moment with a permanent magnet and electromagnet onto the permanent magnet in the robot. However, the relevant controlling means were relatively complicated and were inapplicable to easy control. Mahoney [21] generated a rotating magnetic field by using the permanent magnet at the end of a robot arm with 6 DOFs to drive the movement of the robot with an embedded permanent magnet. Nonetheless, the robot arm may make incorrect actions when it encounters a singular point, which is restricted by the inherent characteristics of the robot arm. The accurate driving control of the CR is consequently affected. Chen [22] simulated the movements of different joints of a CR for esophagus diagnosis and treatment based on ADAMS software and gained the movement characteristic curves of different joints. However, he neglected the hydrodynamic characteristics of the CR in a liquid environment. Tang [23] calculated several movement indexes, such as axial thrust, circumferential resistance moment, and maximum pressure on the inner wall of pipes, of an internal spiral CR in a liquid-solid mixed fluid. The robot still applied an embedded power supply and had limited movement time. $\mathrm{Xu}$ [24] proposed an active $\mathrm{CR}$ equipped with a motor-driven propeller. Control over the direction and speed of this active CR was discussed, but the moving characteristics of the $\mathrm{CR}$ in liquid were not analyzed. $\mathrm{Li}$ [25] conducted a theoretical analysis of stresses on CR in a viscous liquid environment and discussed the dynamic characteristics of CRs with different sizes and shapes. Nevertheless, CR still depended on an embedded battery for power supply.

These research results are mainly related to the moving characteristics and visual transmission characteristics of CRs. However, few studies concerning the self-starting characteristics of CRs exist. In particular, experimental studies related to axial thrust, steady speed, and self-starting speeds under different viscosities are rare. In the current study, a spatial magnetic field distribution model of the master permanent magnet was constructed, and the coupling characteristics between the master and slave permanent magnets were analyzed. The total magnetic induction intensity of the master permanent magnet at any space point was calculated in accordance with the Biot-Savart law. The self-starting characteristics of a MCSCR were analyzed on the basis of the relative rotating movement principle between the master and slave permanent magnets. The relations among self-starting rotating speed, fluid viscosity, and axial thrust were determined. The theoretical analysis and experimental results show that self-starting rotating speed and steady speed are positively related to fluid viscosity, and axial thrust increases gradually as the selfstarting rotating speed increases. The driving flexibility of the MCSCR can be improved significantly. These characteristics offer an effective means for flexible and simple control of the MCSCR. This study lays foundations for accurate and rapid diagnosis and treatment in the entire digestive tract.

The remainder of this study is organized as follows. Section 3 describes the structure of the MCSCR and the magnetic field-driving principle. A magnetic field distribution model and a self-starting characteristic model of the MCSCR are constructed. Section 4 presents the theoretical calculations of fluid viscosity and axial thrust based on the designed models and an analysis on steady speed. The self-starting characteristics of the MCSCR under different fluid viscosities are disclosed. Section 5 shows the conclusions.

\section{Methodology}

\subsection{Structure of the MCSCR and magnetic field-driving principle}

The structure of the MCSCR is shown in Fig. 1. It is composed of an external spiral ribbed sheet, an internal NdFeB permanent magnet (Material designation: N50), and two end covers.

For the convenience of driving, the internal $\mathrm{NdFeB}$ permanent magnet applies the radial magnetization mode (Fig. 2). The NdFeB radial permanent magnet is used as an internal driver, and it forms a coupling driving effect with the external spiral magnetic field. The $\mathrm{NdFeB}$ permanent magnet has the characteristics of high energy density, high 
residual magnetism, and strong stability. The radial magnetization assures that the moving direction of the MCSCR is in accordance with the direction of intestinal tract. This condition is convenient for controlling over the moving directions of the robot and thus strengthens the traveling ability of the MCSCR.

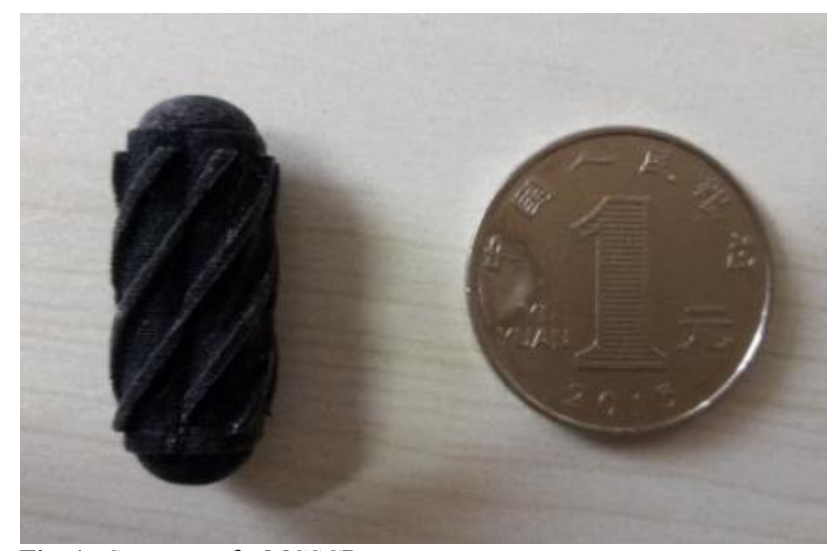

Fig. 1. Structure of a MCSCR

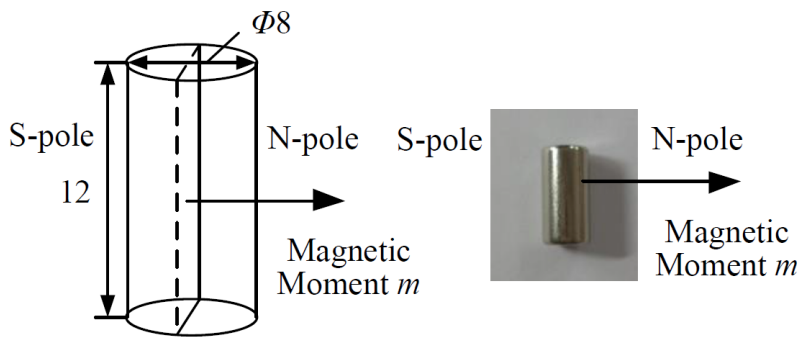

Fig. 2. Radial magnetization of the $\mathrm{NdFeB}$ permanent magnet and a permanent magnet

The theory of magnetism states that $\boldsymbol{B}=\mu_{0} \boldsymbol{H}+\boldsymbol{J}$, where $\boldsymbol{J}$ is the intensity of polarization of the permanent magnet, $\boldsymbol{H}$ is the magnetic field intensity in media, and $\boldsymbol{B}$ is the magnetic induction intensity of media. The permeability of vacuum is $\mu_{0}=4 \pi \times 10^{-7} \mathrm{H} / \mathrm{m}$. The intensity of magnetization per unit volume can be expressed as

$\boldsymbol{M}=\frac{\boldsymbol{J}_{0}}{\mu_{0}}=\frac{\boldsymbol{B}_{r}}{\mu_{0}}$.

Macroscopically, a magnetic charge exists on the circumferential surface along the radial magnetized $\mathrm{NdFeB}$ permanent magnet. The $\mathrm{N}$ and $\mathrm{S}$ poles in the $\mathrm{NdFeB}$ permanent magnet are offset mutually. The magnetic moment of the permanent magnet can be calculated by a surface charge method, which can be expressed as

$m=\sum p_{m}=\sum q_{m} \cdot l_{i}$,

where $p_{m}$ is the dipole moment of the magnet, $q_{m}$ is the magnetic dipole, and $l_{i}(i=1,2,3, \cdots)$ is the displacement vector from $+q_{m}$ to $-q_{m}$.

The NdFeB permanent magnet can be viewed as a superposition of infinite cuboid sheets with different lengths (Fig. 3). The thickness of microelement sheets is denoted by $d x$. Equivalent magnetic charges are generated on the left and right end surfaces of the cuboid, which are viewed as magnetic dipoles. The magnetic moment is $m=2 \int_{-r}^{r} B_{r} \mu_{0}^{-1} L \sqrt{r^{2}-x^{2}} d x$

where $r$ is the radius of permanent magnet, $B_{r}$ is the residual magnetism, $L$ is the length of the permanent magnet, and the $\mathrm{NdFeB}$ permanent magnet size is $\Phi 8 \times 12 \mathrm{~mm}$. Therefore, the magnetic moment of the $\mathrm{NdFeB}$ permanent magnet is $0.7008 \mathrm{~A} \cdot \mathrm{m}^{2}$.

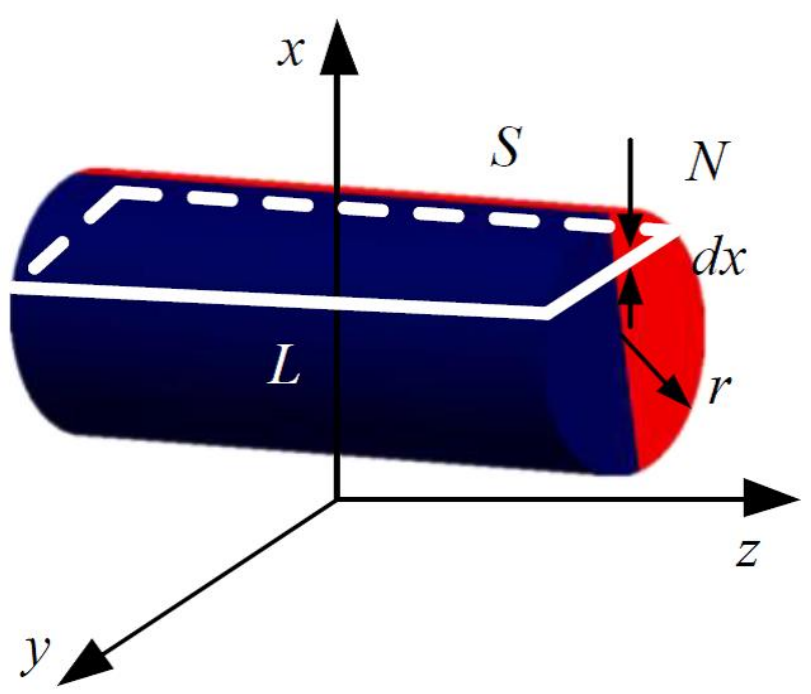

Fig. 3. Microelement diagram of magnetic moment of the permanent magnet

Manufacturing a MCSCR with traditional mechanical processing technique is relatively difficult and requires a high cost; thus, 3D printing was applied to print parts of a MCSCR, which were then assembled. Resin materials, which have the characteristics of toxic free, small density, and light weight, were applied in $3 \mathrm{D}$ printing. The processing accuracy could reach $0.01 \mathrm{~mm}$. These features conform to the design requirements of a robot in this study completely. The main structural parameters of a MCSCR are listed in Table 1. Structural size, including a diameter of 12 $\mathrm{mm}$ and a length of $26 \mathrm{~mm}$, was designed according to the commercialized Pillcam series of CRs; this way meets the clinical requirements on size.

Table 1. Main structural parameters of a MCSCR

\begin{tabular}{c|c}
\hline Parameters & Numerical value \\
\hline MCSCR size $(\mathrm{mm})$ & $\Phi 12 \times 26$ \\
MCSCR mass $(\mathrm{g})$ & 8 \\
NdFeB size $(\mathrm{mm})$ & $\Phi 8 \times 12$ \\
Spiral ribbed height $h_{a}(\mathrm{~mm})$ & 1 \\
Helix angle $\varphi\left({ }^{\circ}\right)$ & 45 \\
Length of tile $L_{c}(\mathrm{~mm})$ & 23 \\
\hline
\end{tabular}

The MCSCR is driven by a portable electromagnetic propulsion device with dynamic rotating magnetic field. A dynamic rotating magnetic field is formed by rotation of the permanent magnet at the end of the output axis of a stepping motor. This magnetic field forms a coupling effect with the internal permanent magnet to drive rotating starts of the MCSCR. The MCSCR has a simple structure and can well adapt to routing inspection in digestive tract and similar environments in human bodies. In addition, the MCSCR can suspend and move forward in the intestinal tract in a liquid environment with high viscosity. The MCSCR avoids 
contact with the intestinal tract and assures safety of internal soft tissues. The rotating and moving directions of the MCSCR are shown in Fig. 4.

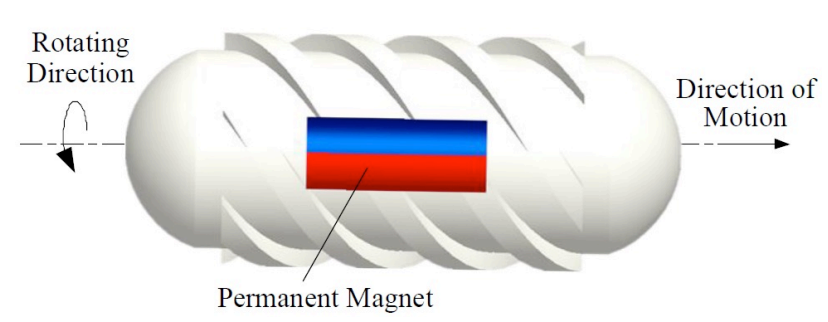

Fig. 4. Moving and rotating directions of a MCSCR

$F_{\text {buo }}=\rho_{L} V_{L} g$ and $G_{\text {rob }}=\rho_{R} V_{R} g$ when the robot is suspending in liquid to prevent direct contact between the robot and intestinal tract and allow the self-suspension of the robot in positioning scanning. $F_{\text {buo }}$ is the buoyancy of the MCSCR, $\rho_{L}$ is the density of fluid, $V_{L}$ is the volume of displaced fluid, $G_{\text {rob }}$ is the gravity of the MCSCR, $\rho_{R}$ is the density of robot materials, $V_{R}$ is the volume of the MCSCR, and $g$ is the acceleration of gravity. The condition for floating of a MCSCR is

$$
\begin{aligned}
& \rho_{R}>\rho_{L}, \text { sinking, } G_{\text {rob }}>F_{\text {buo }} ; \\
& \rho_{R}=\rho_{L}, \text { suspending, } G_{\text {rob }}=F_{\text {buo }} ; \\
& \rho_{R}<\rho_{L}, \text { floating, } G_{\text {rob }}<F_{\text {buo }} .
\end{aligned}
$$

On the basis of the above-mentioned analysis, fluid density shall be equal to robot density to ensure its suspension in the fluid. Appropriate 3D printing materials and fluid viscosity are important factors that assure suspension of the MCSCR.

\subsection{Space magnetic field distribution model of the master permanent magnet}

A portable electromagnetic propulsion device with dynamic rotating magnetic field was designed for driving control of a MCSCR (Fig. 5). A dynamic rotating magnetic field is formed by spirally overlaying two strips of master permanent magnets that are connected with the output axis at the end of a stepping motor, which forms a coupling effect with the slave permanent magnet in the robot. The robot and the external magnetic field rotate synchronously. The MCSCR moves forward and backward in the environment filled with viscous liquid due to rotating effect of the slave permanent magnet. The MCSCR is driven by the speedadjusting function of a stepping motor. This feature can realize continuous stepwise speed adjustment and accurate driving control.

In accordance with the Biot-Savart law, the magnetic induction intensity microelement formed by the master permanent magnet at any space point $P(x, y, z)$ is

$$
d \boldsymbol{B}=\frac{\mu_{0}}{4 \pi} \frac{I d l \times\left(r-r^{\prime}\right)}{\left|r-r^{\prime}\right|^{3}},
$$

where $r$ is the radius vector of the source point $\left(x_{0}, y_{0}, z_{0}\right)$, $r^{\prime}$ is the radius vector of the field point $P(x, y, z), I$ is the current intensity of an internal loop in the permanent magnet, and $I d l$ is a microelement of current intensity. Thus, the total magnetic induction intensity at point $P$ is

$$
\boldsymbol{B}=\frac{\mu_{0}}{4 \pi} \int_{l} \frac{I d l \times\left(r-r^{\prime}\right)}{\left|r-r^{\prime}\right|^{3}} .
$$

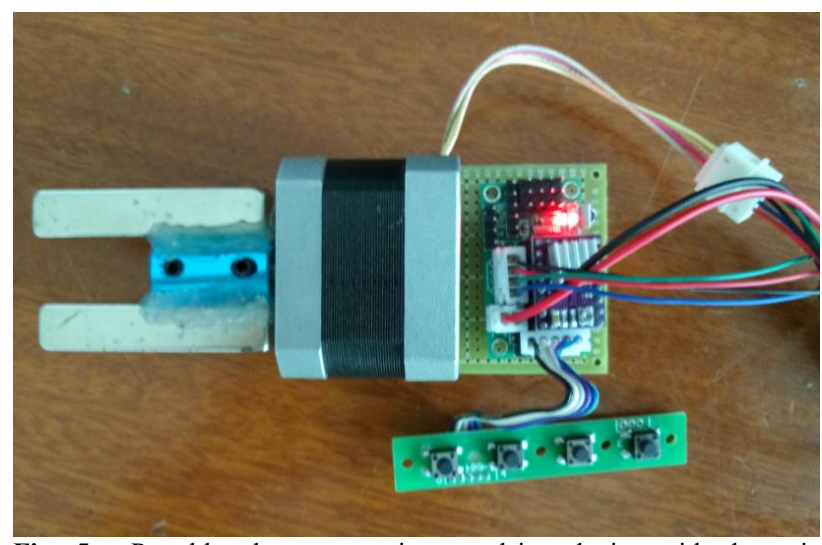

Fig. 5. Portable electromagnetic propulsion device with dynamic rotating magnetic field

The length, width, and height of the master permanent magnet are supposed to be $a, b$, and $c$, respectively (Fig. 6). The magnetic field of the master permanent magnet at any point $P$ in the space is excited by the closed current loop $E F G H E$ on the permanent magnet surface. $Q\left(x_{0}, y_{0}, z_{0}\right)$ is regarded as a point on the surface of the master permanent magnet, and a thin-layered current loop $E^{\prime} F^{\prime} G^{\prime} H^{\prime} E^{\prime}$ along the thickness of the master permanent magnet is selected. The infinitesimal element thickness is $d z_{0}$, and the intensity is $I=J_{s} d z$, where $J_{s}$ is the magnetized current density.

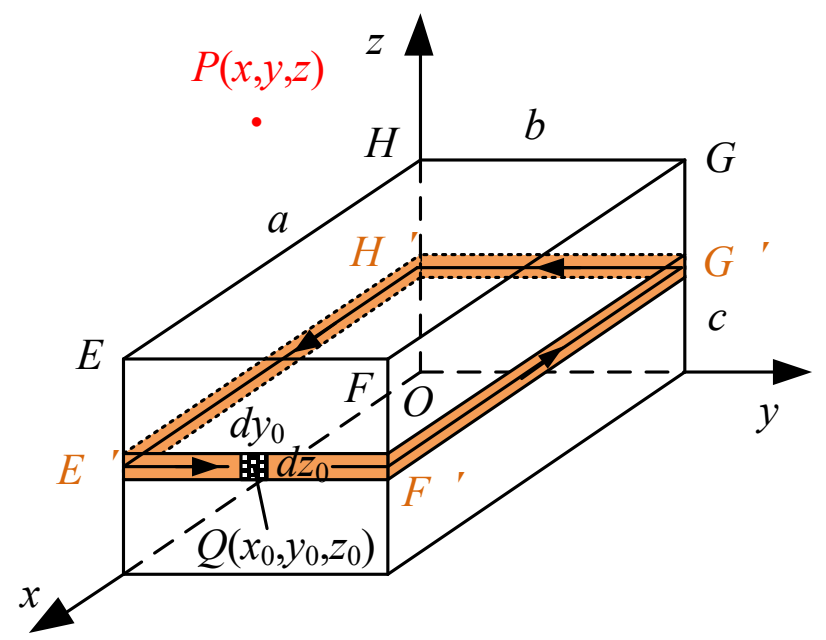

Fig. 6. Spatial distribution of the magnetic induction intensity of the master permanent magnet

The magnetic induction intensity of the current loop at any space point $P$ is $d \boldsymbol{B}$. Therefore, the total magnetic induction intensity generated by a thin-layer current loop is

$\boldsymbol{B}=\int_{0}^{c} d \boldsymbol{B}_{x} \boldsymbol{i}+d \boldsymbol{B}_{y} \boldsymbol{j}+d \boldsymbol{B}_{z} \boldsymbol{k}$,

where $d \boldsymbol{B}_{x}, d \boldsymbol{B}_{y}$, and $d \boldsymbol{B}_{z}$ are the components of the magnetic induction intensity along $x, y$, and $z$, respectively. 
In accordance with the superposition principle of magnetic vectors, vectors of magnetic induction intensities, which are produced by four current segments $\left(E^{\prime} F^{\prime}, F^{\prime} G^{\prime}, G^{\prime} H^{\prime}\right.$, and $\left.H^{\prime} E^{\prime}\right)$ at point $P$, are superposed; this way acquires the total magnetic induction intensity of the master permanent magnet at any space point $P(x, y, z)$.

$$
\left\{\begin{aligned}
\boldsymbol{B}_{x}= & \frac{R}{2}[\mathrm{M}(x, b-y, z)+\mathrm{M}(x, y, z) \\
& -\mathrm{M}(a-x, b-y, z)-\mathrm{M}(\mathrm{a}-x, y, z)] \\
\boldsymbol{B}_{y}= & \frac{R}{2}[\mathrm{M}(y, a-x, z)+\mathrm{M}(y, x, z) \\
& -\mathrm{M}(b-y, a-x, z)-\mathrm{M}(b-y, x, z)], \\
\boldsymbol{B}_{z}= & -R[\mathrm{~N}(a-x, b-y, z)+\mathrm{N}(x, b-y, z) \\
& +\mathrm{N}(a-x, y, z)+\mathrm{N}(x, y, z) \\
& +\mathrm{N}(b-y, a-x, z)+\mathrm{N}(y, a-x, z) \\
& +\mathrm{N}(b-y, x, z)+\mathrm{N}(y, x, z)]
\end{aligned}\right.
$$

where

$$
\mathrm{M}\left(\alpha_{1}, \alpha_{2}, \alpha_{3}\right)=\ln \frac{\left[\alpha_{1}^{2}+\alpha_{2}^{2}+\left(\alpha_{3}-z_{0}\right)^{2}\right]^{\frac{1}{2}}-\alpha_{2}}{\left[\alpha_{1}^{2}+\alpha_{2}^{2}+\left(\alpha_{3}-z_{0}\right)^{2}\right]^{\frac{1}{2}}+\alpha_{2}},
$$$$
\mathrm{N}\left(\beta_{1}, \beta_{2}, \beta_{3}\right)=\frac{\beta_{1}}{\beta_{2}} \frac{\left(\beta_{3}-z_{0}\right)}{\left[\beta_{1}^{2}+\beta_{2}^{2}+\left(\beta_{3}-z_{0}\right)\right]} \text {, and }
$$$$
R=\frac{\mu_{0} J_{s}}{4 \pi}
$$

\subsection{Coupling magnetic moment}

Magnetic coupling theory [26] indicates that the coupling magnetic moment between the external master and internal slave permanent magnets in the robot is perpendicular to the plane formed by the magnetic moment vector $(m)$ of the slave permanent magnet and the magnetic vector $(B)$ of the rotating magnetic field. The coupling magnetic moment is

$$
T_{c}=\boldsymbol{m} \times \boldsymbol{B} .
$$

The fluid resistance moment in the pipe against the movement of the MCSCR is zero at stating moment or stopping state because of the rotating magnetic field of the master permanent magnet. With the increase in rotating speed in the rotating magnetic field, the fluid in the pipe may generate a moment of resistance against the rotation of the MCSCR. As a result, the slave and master permanent magnets cannot make completely synchronous rotation. Instead, a hysteresis angle is formed, which refers to a slip angle of $\delta$. When the axis of the MCSCR is parallel to the axis of the rotating magnetic vector, Eq. (8) can be rewritten as

$$
T_{c}=\boldsymbol{m} \cdot \boldsymbol{B} \cdot \sin \delta,
$$

where $\delta$ is the slip angle between the rotating magnetic vector of the master permanent magnet and the magnetic moment vector of the slave permanent magnet.

One cycle of the slave permanent magnet in the MCSCR, which is driven by the counterclockwise rotation of the external master permanent magnet from position (1) to position (5), is shown in Fig. 7. At initial starting point, $\mathrm{N}$ pole of the slave permanent magnet and $\mathrm{S}$ pole of the master permanent magnet are relatively static. Under this circumstance, the slip angle is zero (Fig. 7(1)). With the increase in speed of the master permanent magnet, the slave permanent magnet is driven to rotate by the coupling magnetic moment. The slave permanent magnet has a hysteresis of one constant slip angle $C$ in relative to the master permanent magnet due to retardation of the MCSCR in a large-viscosity pipe (Fig. 7(2)). When a steady rotating speed is reached, $C$ will be kept until deceleration and stopping. The rest rotating states are shown in Figs. 7(3), (4), and (5).

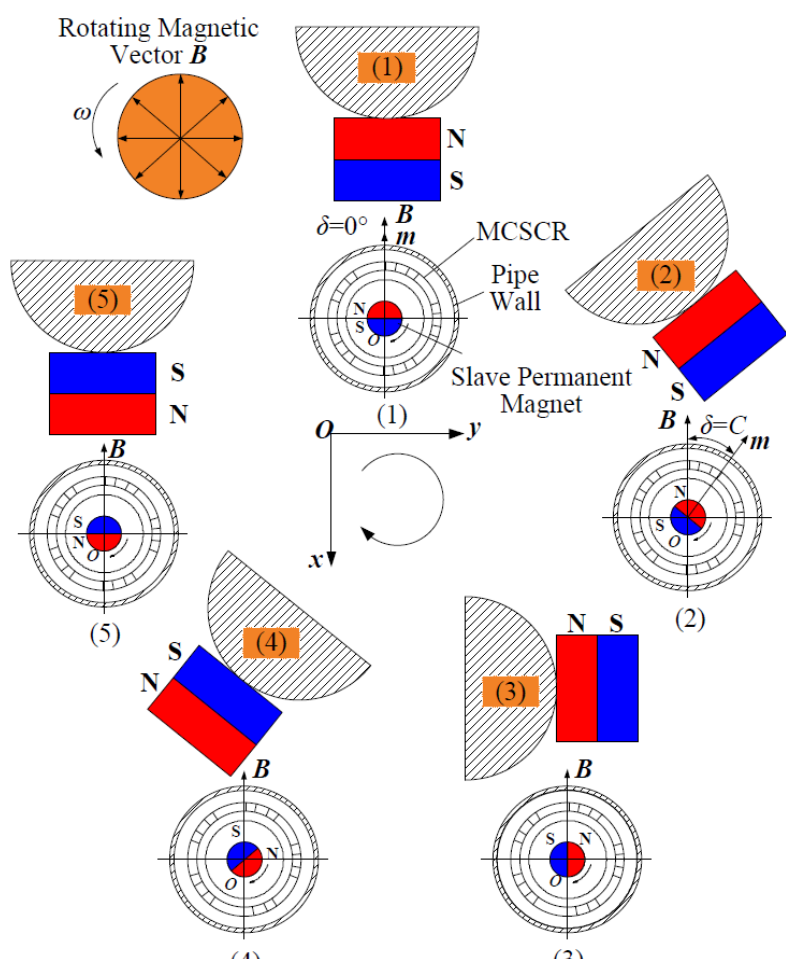

Fig. 7. Relative rotating principle between the master and slave permanent magnets

In the process from stating moment to steady rotation of the MCSCR, the coupling magnetic moment increases with the increase in slip angle. When the fluid resistance moment is smaller than the coupling magnetic moment,

$$
T_{f}<T_{c} .
$$

As the MCSCR accelerates to rotate gradually, the fluid resistance moment is increased accordingly. When the fluid resistance moment is equal to the coupling magnetic moment,

$T_{f}=T_{c}$

The MCSCR reaches a steady speed. Under this circumstance, the slave permanent magnet in the MCSCR and the rotating magnetic field rotate synchronously, whereas the slip angle and fluid resistance moment are kept constant. At deceleration of the rotating magnetic field, the fluid resistance moment is higher than the coupling magnetic moment, and

$$
T_{f}>T_{c} .
$$


The hydrodynamic pressure of the MCSCR is decreased, which decreases the fluid resistance moment of large viscosity against the MCSCR gradually. The MCSCR can be driven inversely by portable electromagnetic propulsion device with dynamic rotating magnetic field when adjusting the counterclockwise rotation of the stepping motor. In this way, the MCSCR makes retreated movement.

\subsection{Self-starting characteristics}

In a pipe filled with large-viscosity fluid, the external surface of a MCSCR is adhered with spiral ribs to facilitate backward outflow of the fluid along the axis of the MCSCR due to self-rotating of the robot. The MCSCR is driven by the shearing force of the fluid and a counter-acting force of pressure to move forward. In other words, if the axial thrust $F_{a x i}$ and the resistance of fluid against the MCSCR is $F_{r e s}$, then the resultant force on the MCSCR is $F=F_{a x i}-F_{r e s}$. The stress and moment distribution of the MCSCR are shown in Fig. 8.

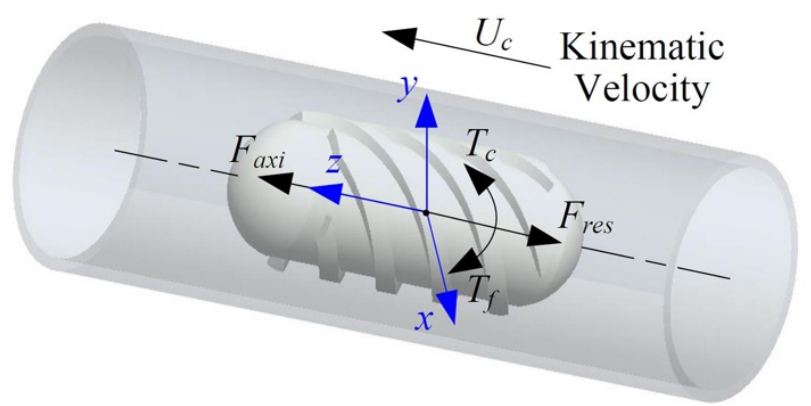

Fig. 8. Dynamic equilibrium of a MCSCR

At the initial starting, if $F_{r e s}>F_{a x i}$, then the MCSCR begins to rotate rapidly from the static position when $F<0$. When the rotating speed of the MCSCR reaches the critical rotating speed of self-starting, $F_{r e s}=F_{a x i}$. At this moment, $F=0$. When the MCSCR continues to accelerate until $F_{r e s}=F_{a x i}$ is reached again, it will achieve the stress equilibrium along the axial direction, and the axial thrust is equal to the resistance of fluid. Therefore, the MCSCR moves forward at a steady speed.

$F_{a x i}=F_{r e s}$.

In this case, the constant feeding speed of the MCSCR is $U_{c}$. Fluid resistance is related to the shape, speed, and incident flow of a robot and the properties of the fluid (e.g., viscosity and compression). Therefore, the resistance of fluid against the MCSCR can be expressed as

$$
F_{r e s}=C_{D} \frac{\rho U_{c}^{2}}{2} A
$$

where $C_{D}$ is a resistance coefficient and $A$ is the projection area of incident flow of the MCSCR, that is, the resistance area.

When the MCSCR moves in fluid, the fluid resistance is directly related to the Reynolds number and external shape of the robot. When the Reynolds number is relatively low, viscosity, which is related to axial thrust, is the main influencing factor, and effects of inertial force are neglected. The Reynolds number in a round pipe is
$R e=\frac{U_{c} D \rho}{\mu}$,

where $D$ is the diameter of the pipe, $\rho$ is the density of fluid, and $\mu$ is the dynamic viscosity of fluid. The diameter of the round pipe is very small and the dynamic viscosity is $\mu=0.5 \mathrm{~Pa} \cdot \mathrm{s}$ due to the low movement velocity of MCSCR. Therefore, $R e<1$. On this basis, the resistance of viscous fluid against the MCSCR can be deduced from the Stokes formula as

$F_{\text {res }}=3 \pi \mu d U_{c}$,

where $d$ is the diameter of the hemispherical head of the MCSCR.

From the combination of Eqs. (14), (15), and (16), the resistance coefficient can be gained as

$C_{D}=\frac{24}{R e}$

Finally, resistance of fluid against the MCSCR can be gained as

$F_{r e s}=\frac{24}{\operatorname{Re}} \frac{\rho U_{c}^{2}}{2} A$.

The dynamic self-starting process of a MCSCR is shown in Fig. 9. First, when the rotating speed of the external magnetic field is low and is lower than the self-starting of the MCSCR, the MCSCR only spins around but cannot move forward, which is caused by the retardation of viscous liquid. Later, the rotating speed of the MCSCR increases with the increase in the rotating speed of the external magnetic field, and the MCSCR begins to move forward. This rotating speed of the external magnetic field is called self-starting rotating speed. Subsequently, the MCSCR can move stably along the center of skewed pipe after a short period of steady adjustment because of the influence of the dead load and external magnetic field (Fig. 9(c)).
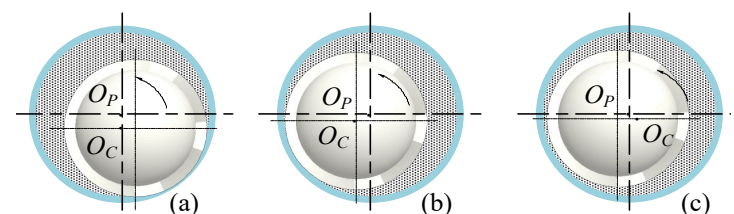

Fig. 9. Dynamic self-starting process of a MCSCR. (a) Static state. (b) Starting moment. (c) Stable moving

\subsection{Experiment}

A MCSCR and a portable electromagnetic propulsion device with dynamic rotating magnetic field in Table 1 were developed to verify the accuracy of the above-mentioned theoretical analysis and self-starting rotating speed of the robot (Fig. 10). A rotating magnetic field control experiment was conducted.

For the convenience of comparison, the experiment was performed in a transparent acrylic glass tube with a MCSCR. The inner diameter of this acrylic glass tube is $19 \mathrm{~mm}$, and its two ends have nonclosed connection with PVC bends to assure natural mobility of liquid in the tube. The acrylic glass tube was filled with the mixing liquid formed by 
viscosity-varying water and glycerinum at different proportions based on the Grunberg-Nissan method [27] for hydrodynamic pressure test under different viscosities. The mole numbers of water and glycerinum, which were corrected by the Brookfield Viscometer, are shown in Table 2 .

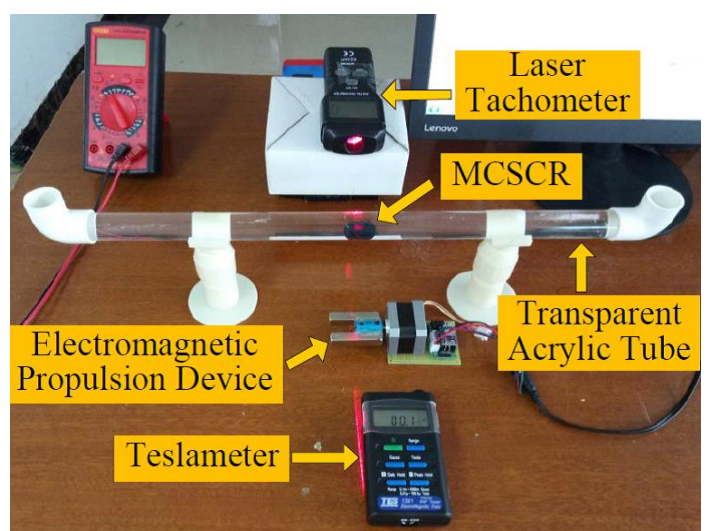

Fig. 10. Experimental system for portable dynamic rotating magnetic field control

Table 2. Mole number under different fluid viscosities

\begin{tabular}{l|l|l|l|l|l|l}
\hline$\mu(\mathrm{Pa} \cdot \mathrm{s})$ & 0.1 & 0.2 & 0.3 & 0.4 & 0.5 & 0.6 \\
\hline Water(mol) & 0.46 & 0.35 & 0.22 & 0.21 & 0.15 & 0.11 \\
Glycerinum(mol) & 0.54 & 0.65 & 0.78 & 0.79 & 0.85 & 0.89 \\
\hline
\end{tabular}

The measurement of axial thrust is shown in Fig. 11. A fine line was extended from the MCSCR tail to connect with the digital push and pull tester (VICTOR 10N). The numerical value of the axial thrust of a MCSCR, which was measured by a digital push-and-pull tester, can be displayed on the tester or a computer.

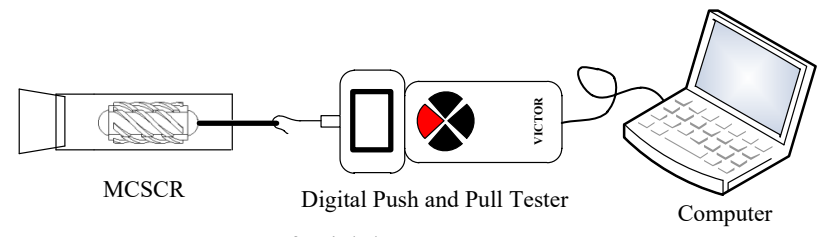

Fig. 11. Measurement of axial thrust

The self-starting rotating speed of a MCSCR in a transparent acrylic glass tube filled with different fluid viscosities was tested (Fig. 12). White reflective paint was coated on the side of the MCSCR, and the rotating speed was measured by a laser rotating speed meter (VIC6234P).

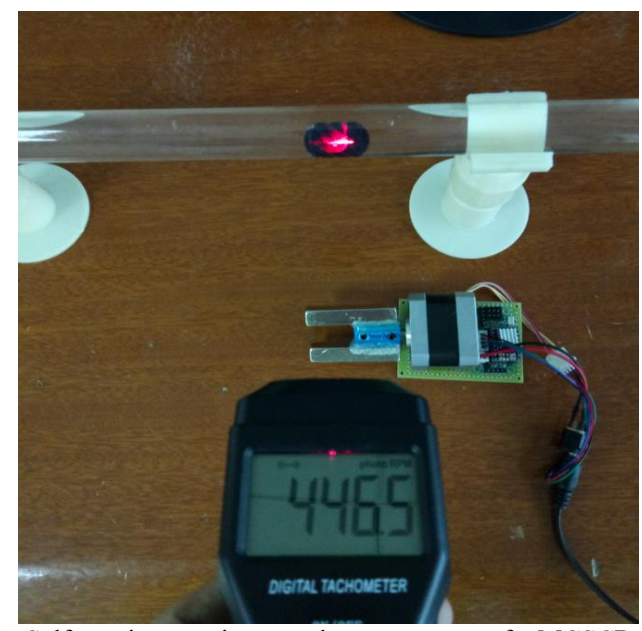

Fig. 12. Self-starting rotating speed measurement of a MCSCR

\section{Result analysis and discussion}

4.1 Influences of fluid viscosity on axial thrust and steady speed

The simulation curves of the axial thrust and steady speed of the MCSCR under different fluid viscosities are shown in Fig. 13. Given a fixed fluid viscosity, axial thrust is positively correlated with steady speed. When fluid viscosity increases, the axial thrust is increased accordingly.

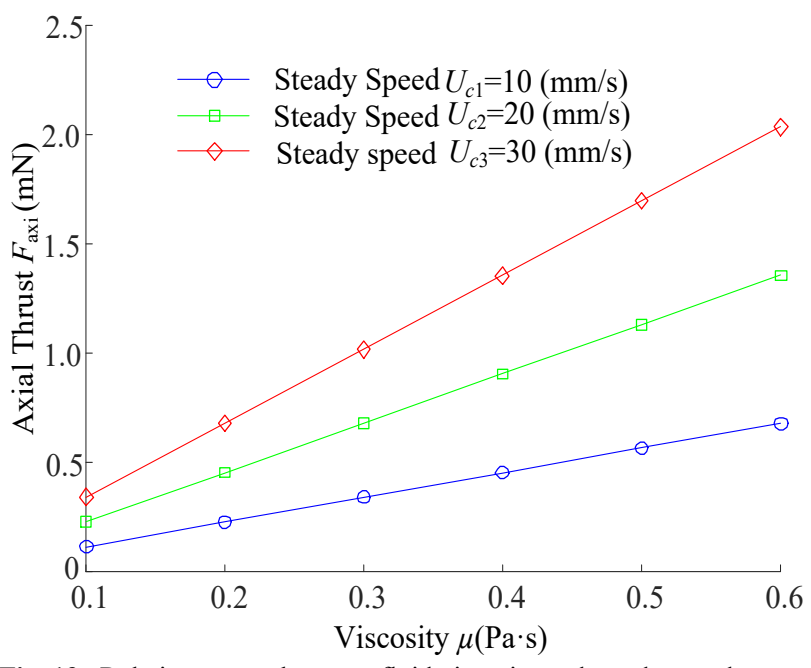

Fig. 13. Relation curves between fluid viscosity and steady speed

The simulation relation curves between axial thrust and fluid viscosity are shown in Fig. 14. Given the same fluid viscosity, the steady speed is proportional to axial thrust. When the axial thrust is fixed, the steady speed is negatively related to fluid viscosity. In summary, fluid viscosity is vital to the movement of a MCSCR. When the axial thrust is $0.5 \mathrm{mN}$, the steady speed becomes a constant at $0.6 \mathrm{~Pa} \cdot \mathrm{s}$ of fluid viscosity. With the continuous increase in viscosity, the steady speed changes slightly.

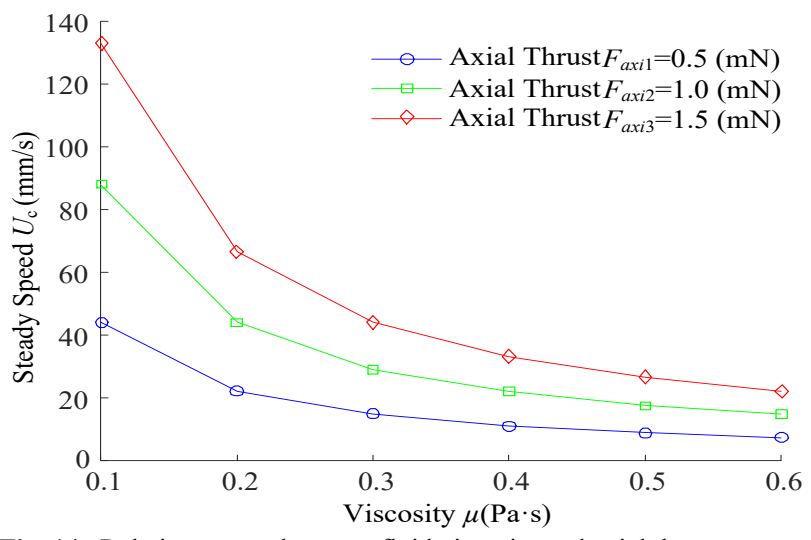

Fig. 14. Relation curves between fluid viscosity and axial thrust

4.2 Influences of steady speed and axial thrust on selfstarting rotating speed

The simulated relation curves between the self-starting rotating and steady speeds of a MCSCR under different fluid viscosities $(\mu=0.1,0.3,0.5 \mathrm{~Pa} \cdot \mathrm{s})$ are shown in Fig. 15 . The intersection point between curves and the $x$-axis is the corresponding self-starting rotating speed. The self-starting rotating speed of a MCSCR is negatively correlated with fluid viscosity, whereas the rotating speed of a started 
MCSCR is positively related to steady speed. Fig. 15 depicts that self-starting rotating speeds are 52,71 , and $91 \mathrm{r} / \mathrm{min}$ when $\mu=0.1,0.3,0.5 \mathrm{~Pa} \cdot \mathrm{s}$, respectively.

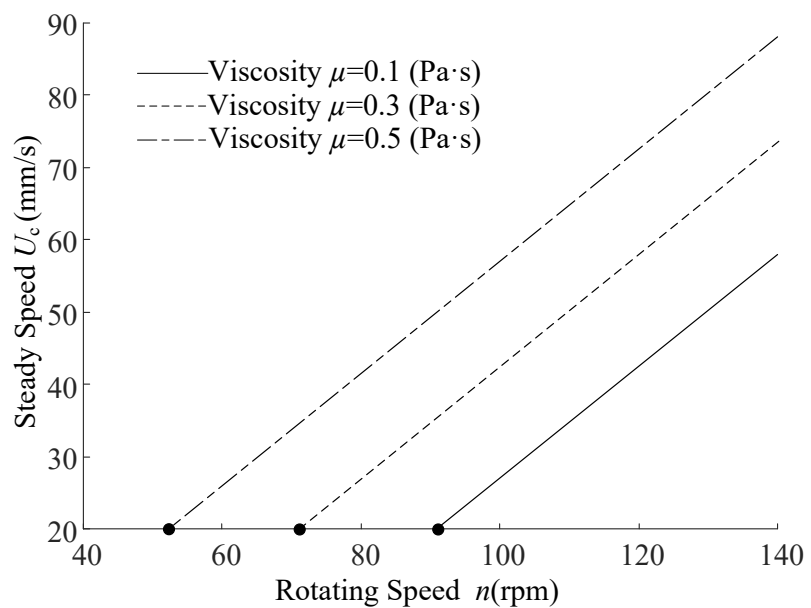

Fig. 15. Relation curves between self-starting rotating and steady speeds

The simulated relation curve between self-starting rotating speed and fluid viscosity is shown in Fig. 16. The intersection point between curves and the $x$-axis is the corresponding self-starting rotating speed. Self-starting rotating speed decreases gradually with the increase in fluid viscosity. The MCSCR has to overcome a strong resistance when a large-viscosity fluid exists. High viscosity causes strong resistance of fluid and a strong axial thrust that is needed.

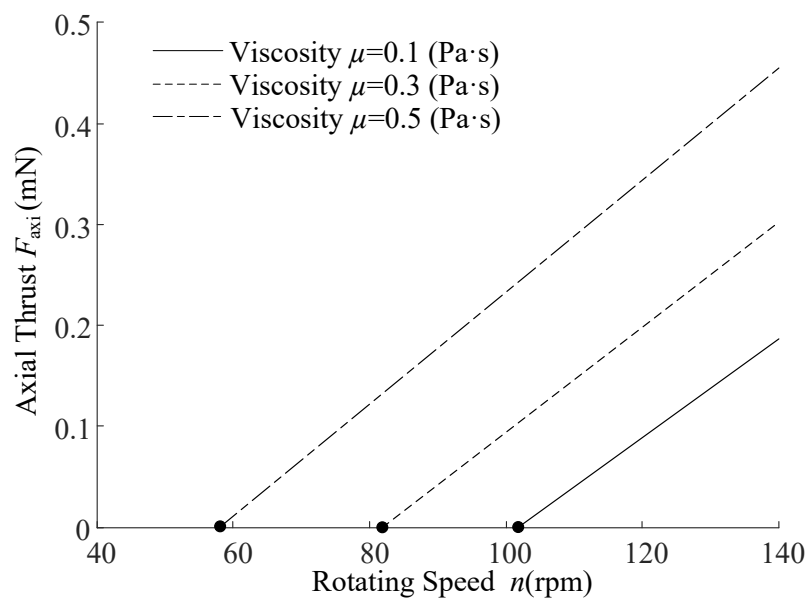

Fig. 16. Relation curves between self-starting rotating speed and fluid viscosity

\subsection{Experimental results of self-starting rotating speed}

Comparison between theoretical simulation and experimental results on the self-starting rotating speed of a MCSCR is shown in Fig. 17. The experimental results conform to the theoretical analysis results, and this conformity verifies the accuracy of the self-starting characteristic model of the above-mentioned fluids.

The measured self-starting rotating speeds are shown in Table 3. The self-starting rotating speed of a MCSCR increases with the increase in fluid viscosities, and the minimum is achieved $(85 \mathrm{r} / \mathrm{min})$ when the fluid viscosity is $0.1 \mathrm{~Pa} \cdot \mathrm{s}$.

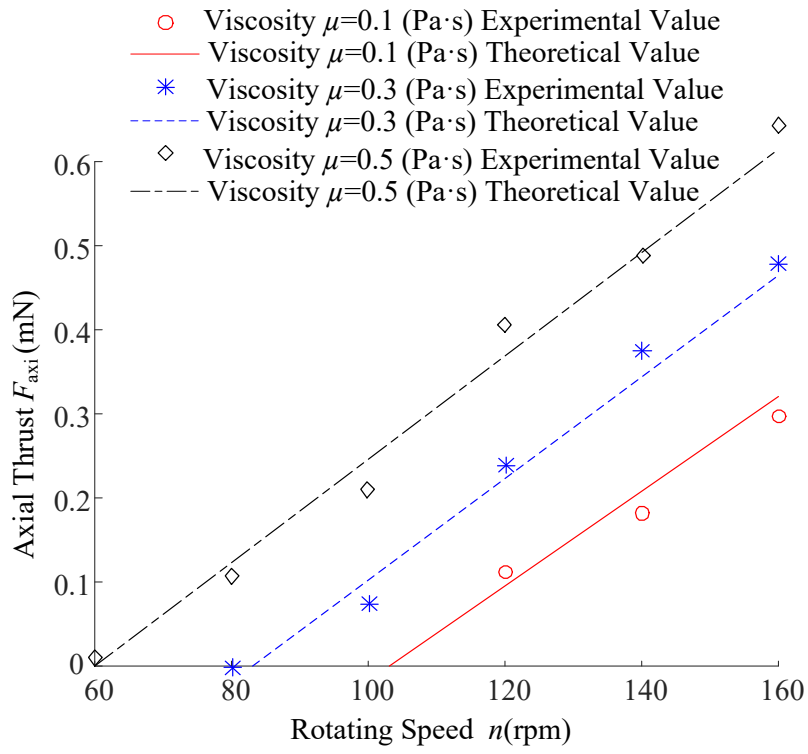

Fig. 17. Experimental curves of self-starting rotating speed, axial thrust, and fluid viscosity

Table. 3. Self-starting rotating speed under different fluid viscosities

\begin{tabular}{l|l|l|l|l|l|l}
\hline $\begin{array}{l}\text { Fluid viscosities } \\
\mu(\mathrm{Pa} \cdot \mathrm{s})\end{array}$ & 0.1 & 0.2 & 0.3 & 0.4 & 0.5 & 0.6 \\
\hline $\begin{array}{l}\text { Self-starting } \\
\text { rotating speed } \\
n(\mathrm{rpm})\end{array}$ & 85 & 133 & 171 & 198 & 216 & 288 \\
\hline
\end{tabular}

The relationship between coupling magnetic moment and self-starting distance was tested to determine the maximum self-starting distance to drive a MCSCR. The results are shown in Fig. 18. With the increase in distance to a MCSCR, the coupling magnetic moment decreases gradually. When the master permanent magnet is $29 \mathrm{~mm}$ away from the MCSCR, the maximum coupling magnetic moment reaches the minimum $(0.1 \mathrm{mN} \cdot \mathrm{m})$.

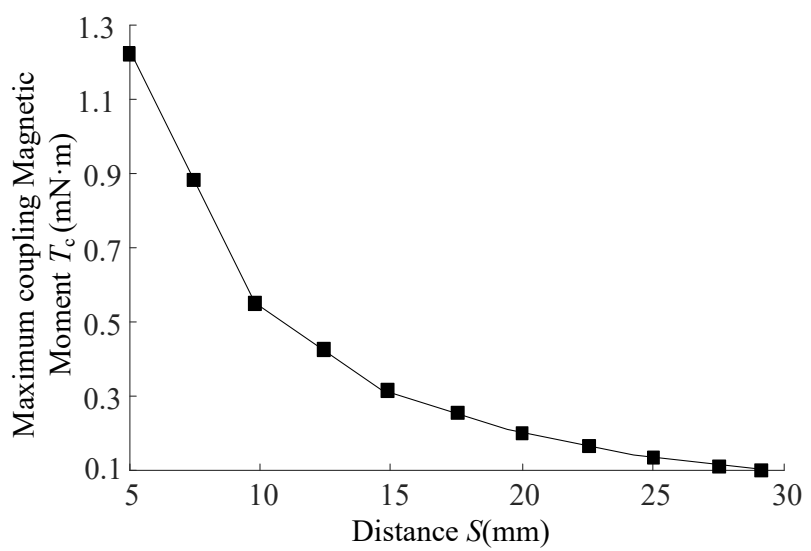

Fig. 18. Relation curves between the maximum coupling magnetic moment and distance to the MCSCR

\section{Conclusions}

For simple control over a MCSCR and disclosing the relationship between the self-starting characteristics of the MCSCR and fluid viscosity, a portable electromagnetic propulsion device with dynamic rotating magnetic field and a MCSCR were developed. A spatial magnetic field distribution model of permanent magnet was constructed. A theoretical analysis and an experimental study on the coupling characteristics between the master and slave 
permanent magnets and the self-starting characteristics of a MCSCR were performed. The following conclusions could be drawn:

(1) The swimming speed of a MCSCR can be adjusted by controlling the rotating speed of the master permanent magnet. Fluid viscosity is inversely proportional to the steady speed of a MCSCR. Given the same viscosity, steady speed is positively related to the rotating speed of the MCSCR.

(2) Fluid viscosity is proportional to the axial thrust of a MCSCR. In other words, axial thrust is needed to be high under high fluid viscosity. Axial thrust increases gradually with the increase in steady speed. When the fluid viscosity is $0.1 \mathrm{~Pa} \cdot \mathrm{s}$, the self-starting rotating speed is the lowest at $85 \mathrm{r} / \mathrm{min}$.

(3) The self-starting rotating speed of a MCSCR increases with the increase in fluid viscosity. Axial thrust is also positively related to rotating speed. Given the same fluid viscosity, the self-starting rotating speed and axial thrust are proportional to steady speed.

(4) The coupling magnetic moment can be adjusted by controlling the distance between the master permanent magnet and the MCSCR. The coupling magnetic moment decreases with the increase in distance. Self-starting of a MCSCR can be controlled by adjusting the self-starting distance between the coupling magnetic moment and the MCSCR. When the master permanent magnet is $29 \mathrm{~mm}$ away from the MCSCR, the maximum coupling magnetic moment reaches the lowest level at $0.1 \mathrm{mN} \cdot \mathrm{m}$.

In this study, relations of the axial thrust, coupling magnetic moment, and steady speed of a MCSCR are disclosed by controlling its self-starting rotating speed under different fluid viscosities. On this basis, driving control of a MCSCR with a low torque and a strong axial thrust can be realized. This study lays a foundation for clinical applications of in vivo MCSCRs for rapid detection with low power consumption. However, without positioning technology of MCSCRs, future studies shall carry out an in vitro experiment in intestinal tract by combining the posture positioning of the MCSCRs and research results in this study to acquire a highly accurate understanding on self-starting characteristics.

\section{Acknowledgements}

This work was supported by the Key Scientific and Technological Project of Henan Province (Grant No. 182102210261)

This is an Open Access article distributed under the terms of the Creative Commons Attribution License

\section{References}

1. Shukla, A., Karki, H., "Application of robotics in onshore oil and gas industry-A review Part I". Robotics and Autonomous Systems, 75, 2016, pp.490-507.

2. Wang, J. C., Wang, Z., Leach, M., Sanghyuk, L., Lim, E. G., "RF characteristics of wireless capsule endoscopy in human body". Journal of Central South University, 23(5), 2016, pp.1198-1207.

3. Jang, J., Lee, J., Lee, K. R., Lee, J., “A four-camera VGA-resolution capsule endoscope system with $80-\mathrm{Mb} / \mathrm{s}$ body channel communication transceiver and sub-centimeter range capsule localization". IEEE Journal of Solid-State Circuits, 54(2), 2019, pp.538-549.

4. Kim, H. M., Yang, S., Kim, J., Park, S., "Active locomotion of a paddling-based capsule endoscope in an in vitro and in vivo experiment (with videos)". Gastrointestinal Endoscopy, 72(2), 2010, pp.381-387.

5. Gao, J., Yan, G., Wang, Z., He, S., "Design and testing of a motorbased capsule robot powered by wireless power transmission". IEEE/ASME Transactions on Mechatronics, 21(2), 2016, pp.683693.

6. Shao, Q., Liu, H., Yang, Z., Wang, H., Li, H., "Design and analysis of micro legged capsule robot”. Robot, 37(2), 2015, pp.246-253.

7. Hong, Y. S., Kim, J. Y., Kwon, Y. C., Song, S. Y., "Preliminary study on capsule endoscopes propelled by variable threads". Biomedical Engineering, 7, 2010, pp.202-207.

8. Yan, Y., Liu, Y., Manfredi, L., Prasad, L., "Modelling of a vibroimpact self-propelled capsule in the small intestine". Nonlinear Dynamics, 96(1), 2019, pp.123-144.

9. Fu, Q., Guo, S., Huang, Q., Hirata, H., "Development and evaluation of novel magnetic actuated microrobot with spiral motion using electromagnetic actuation system". Journal of Medical and Biological Engineering, 36(4), 2016, pp.506-514.

10. Diller, E., Giltinan, J., Lum, G. Z., Ye, Z., "Six-degree-of-freedom magnetic actuation for wireless microrobotic". The International Journal of Robotics Research, 35(1), 2015, pp.114-128.

11. Park, C., Kim, J., Kim, S. J., Yoo, J., "Development of a permanent magnet type micro-robot actuated by external electromagnetic system”. Microsystem Technologies, 21(6), 2015, pp.1257-1265.

12. Fu, Q., Guo, S., "Design and performance evaluation of a novel mechanism with screw jet motion for a hybrid microrobot driven by rotational magnetic field". In: 2016 IEEE International Conference on Mechatronics and Automation, Harbin, China: IEEE, 2016, pp. 2376-2380.
13. Fu, Q., Guo, S., Yamauchi, Y., Hirata, H., "A novel hybrid microrobot using rotational magnetic field for medical applications". Biomedical Microdevices, 17(2), 2015, pp.1-12.

14. Sendoh, M., Sudi, Y., Ishiyama, K., Arai, K. I., "Fabrication of magnetic actuator for use in colon endoscope". IEEE Transactions on Magnetics, 39(5), 2003, pp.3232-3234.

15. Lee, C., Choi, H., Go, G., Jeong, S., "Active locomotive intestinal capsule endoscope (ALICE) system: A prospective feasibility study". IEEE/ASME Transactions on Mechatronics, 20(5), 2014, pp.2067-2074.

16. Guo, S., Liang, N., Guo, J., Liu, P., "Movement characteristics evaluation of the spherical robot actuated by the magnetic field for medical applications". In: 2016 IEEE International Conference on Mechatronics and Automation, Harbin, China: IEEE, 2016, pp.448453.

17. Yang, K., Yan, G., Gao, J., "Design of wireless power supply system for robotic capsule". Beijing Biomedical Engineering, 35(5), 2016, pp.510-515.

18. Zhang, P., "Study on the Structure and Control of Magnetic MultiModular Capsule Robotic System". Master thesis of Tianjin University of Technology, China, 2019, pp.35-43.

19. Salerno, M., Rizzo, R., Sinibaldi, E., Menciassi, A., "Force calculation for localized magnetic driven capsule endoscopes". In: 2013 IEEE International Conference on Robotics and Automation, Karlsruhe, Germany: IEEE, 2013, pp.5354-5359.

20. Gumprecht, J. D. J., Lueth, T. C., Khamesee, M. B., "Navigation of a robotic capsule endoscope with a novel ultrasound tracking system". Microsystem Technologies, 19(9-10), 2013, pp.1415-1423.

21. Mahoney, A. W., Abbott, J. J., "Generating rotating magnetic fields with a single permanent magnet for propulsion of untethered magnetic devices in a lumen". IEEE Transactions on Robotics, 30(2), 2014, pp.411-420.

22. Chen, X., Yu, J., Qian, S., Cao, P., "Dynamic study of capsuledriven robot for esophagus diagnosis and treatment based on ADAMS". Light Industry Machinery, 37(4), 2019, pp.30-34+41.

23. Tang, P., Liang, L., Xu, Y., Chen, B., "Study on characteristics of inner spiral capsule robot in liquid-solid two-phase flow". Lubrication Engineering, 43(9), 2018, pp.62-66.

24. Xu, J., Zhang, Y., Liu, B., Li, J., "An active wireless capsule endoscope microrobot". China Medical Device Information, 21(10), 2015, pp.1-5+33. 
25. Li, X., Yu, J., Qian, S., Cao, P., "Stress model and fluid solid coupling simulation of microcapsule in simulated intestine". Food \&Machinery, 35(2), 2019, pp.7-10.

26. Zhang, Y., Su, Z., Chi, M., Huang, Y., "Magnitude and orientation error correction of a superimposed spatial universal rotating magnetic vector". IEEE Transactions on Magnetics, 52(5), 2016, pp.1-9.
27. Marczak, W., Adamczyk, N., Łezniak, M., "Viscosity of associated mixtures approximated by the grunberg-nissan model". International Journal of Thermophysics, 33(4), 2012, pp.680-691. 